\title{
Characterization of diffraction gratings by use of a tabletop soft-x-ray laser
}

\author{
Max Seminario, Jorge J. Rocca, Ricardo A. Depine, Benny Bach, and Bernie Bach
}

\begin{abstract}
We have demonstrated the use of a high-repetition-rate 46.9-nm tabletop laser to characterize diffraction gratings designed for grazing-incidence operation in the soft-x-ray spectral region. The efficiencies for various diffraction orders were measured as a function of angle of incidence and compared with the results of model simulations. This measurement technique provides benchmarks with which to improve electromagnetic codes used in the design of soft-x-ray diffraction gratings. The results illustrate the potential of compact tabletop soft-x-ray lasers for use as a new tool for characterization of shortwavelength optics at the manufacturer's site. (C) 2001 Optical Society of America

OCIS codes: $340.0340,140.7240,350.2770$.
\end{abstract}

\section{Introduction}

Diffraction gratings designed for efficient operation at wavelengths below $50 \mathrm{~nm}$ are finding increased use in monochromators for synchrotron beam lines and high-order harmonic sources of short-wavelength coherent radiation,1,2 in vacuum spectrographs for plasma diagnostics, and in soft-x-ray interferometers. $^{3,4}$ Accurate measurement of the diffraction efficiency and development of electromagnetic codes for grating design ${ }^{5,6}$ are of significant interest for costeffective manufacturing of gratings for various applications.

The recent advent of compact high-repetition-rate tabletop soft-x-ray lasers with milliwatt average power ${ }^{7,8}$ opens the opportunity for making, in a tabletop setup, a detailed characterization of such diffraction gratings. In this paper we report the use of a highrepetition-rate tabletop laser operating at $46.9 \mathrm{~nm}$ to characterize the diffraction efficiency of two diffraction gratings designed for grazing-incidence opera-

M. Seminario and J. J. Rocca (rocca@engr.colostate.edu) are with the Department of Electrical and Computer Engineering, Colorado State University, Fort Collins, Colorado 80523. R. A. Depine (rdep@df.uba.ar) is with the Grupo de Electromagnetismo Aplicado, Departamento de Física, Facultad de Ciencias Exactas, Universidad de Buenos Aires, Ciudad Universitatia, Pabellón 1, 1428 Buenos Aires, Argentina. B. Bach and B. Bach are with Hyperfine, Incorporated, 4946 North 63rd Street, Boulder, Colorado 80301.

Received 26 September 2000; revised manuscript received 16 April 2001.

0003-6935/01/305539-06\$15.00/0

(C) 2001 Optical Society of America tion at that wavelength. Other schemes for performing these measurements could be implemented with a synchrotron radiation source, a lasercreated plasma, or a discharge-pumped soft-x-ray lamp. However, when measurements at multiple wavelengths are not necessary, the capillary discharge laser offers the advantages of its high average power, highly directional beam, and compact size.

Measurements of the diffraction efficiencies that correspond to various diffraction orders were compared with the simulations of an electromagnetic code. The code is based on a class of theoretical methods known in the literature as differential. ${ }^{9}$ The term "differential" refers to the fact that the rigorous computation of the electromagnetic field diffracted by the grating is reduced in these methods to the resolution of a system of ordinary differential coupled equations.

The rest of this paper is organized as follows: In section 2 we describe the experimental setup used in measuring the diffraction efficiency. In Section 3 we describe the numerical code used to simulate the performance of the gratings, and in Section 4 we compare the experimental and theoretical results.

\section{Experimental Setup and Description of Gratings}

Two gold-coated diffraction gratings designed for operation at $46.9 \mathrm{~nm}$ were characterized. Each grating was ruled on a borosilicate substrate by a diamond ruling engine. The first grating had a ruling of 1800 lines/mm and was blazed at $10.028 \mathrm{deg}$. The second was ruled with a line density of 300 lines/mm at a blaze angle of $1.33 \mathrm{deg}$. This grating was designed to split the beam intensity evenly be- 

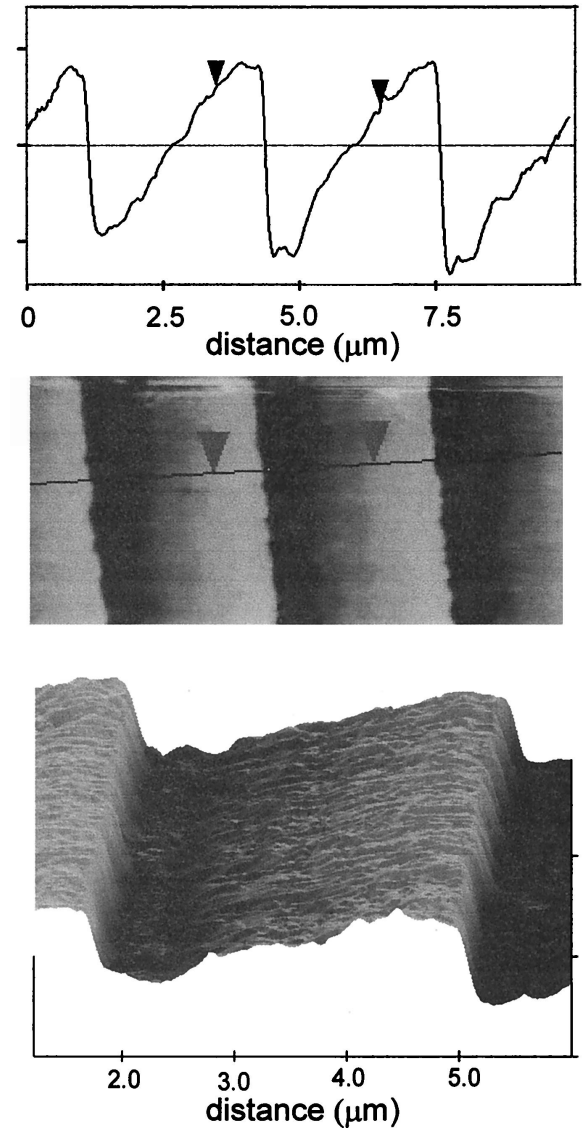

Fig. 1. Atomic-force microscope image of the groove profile corresponding to the $300-$ line $/ \mathrm{mm}$ diffraction grating and (top) trace of the image.

tween the 0 order and the -1 order for use as a beam splitter in an amplitude division soft-x-ray interferometer. ${ }^{3}$ Each grating had a gold coating that was electron-beam evaporated over a chromium layer. The groove profiles were measured with an atomic-force microscope. Figure 1 shows the measured profile of the 300 -line/ $\mathrm{mm}$ grating. The profile can be observed to have a triangular shape, justifying the use of ideal triangular profiles in the model calculations.

Figure 2 is a schematic illustration of the setup with which we measured the diffraction efficiency. The gratings were illuminated with a beam of 46.9-nm radiation produced by a tabletop capillary discharge neonlike argon laser operating at $46.9 \mathrm{~nm}$. The characteristics of this tabletop laser were discussed in recent publications. ${ }^{7,8}$ Laser amplification occurs by collisional excitation of the $3 p{ }^{1} S_{0}-3 s{ }^{1} P_{1}$ transition of the neonlike argon ion in an elongated plasma column that is generated by excitation of a capillary channel with a fast current pulse. The magnetic force of the current pulse rapidly compresses the plasma to form a dense and hot column with a length-to-diameter ratio approaching 1000:1, in which the conditions for amplification by collisional excitation occur near the end of the compression. ${ }^{10}$ To characterize the diffraction gratings, we generated laser pulses with an energy of approximately $0.1 \mathrm{~mJ}$ and pulse widths of $\sim 1.2 \mathrm{~ns}$ at a repetition rate of $1-2 \mathrm{~Hz}$, with an 18.2-cm-long capillary discharge plasma column. The resultant laser beam divergence was $\sim 4.5 \mathrm{mrad}$. The laser that we used is very compact; it occupies an optical table area of only $0.4 \mathrm{~m} \times 1 \mathrm{~m}$, a size comparable with that of many widely used visible and ultraviolet gas lasers. Such a compact size opens the opportunity for conducting this type of measurement at the grating manufacturer's site.

The measurements were conducted in a vacuum chamber placed $\sim 1.5 \mathrm{~m}$ from the exit of the laser. The diffraction gratings were mounted upon the axis of a rotational stage that allowed for selection of the angle of incidence. The intensity of the diffracted beam was recorded with a vacuum photodiode (Photodiode B in Fig. 2). This detector was mounted upon a lever arm driven by a stepper motor that allowed, without breaking vacuum, positioning of the photodiode at each of the angles that corresponds to a diffracted order. To allow for measurements at large angles of incidence we reduced the laser beam size by placing a 1-mm-diameter pinhole at the en-

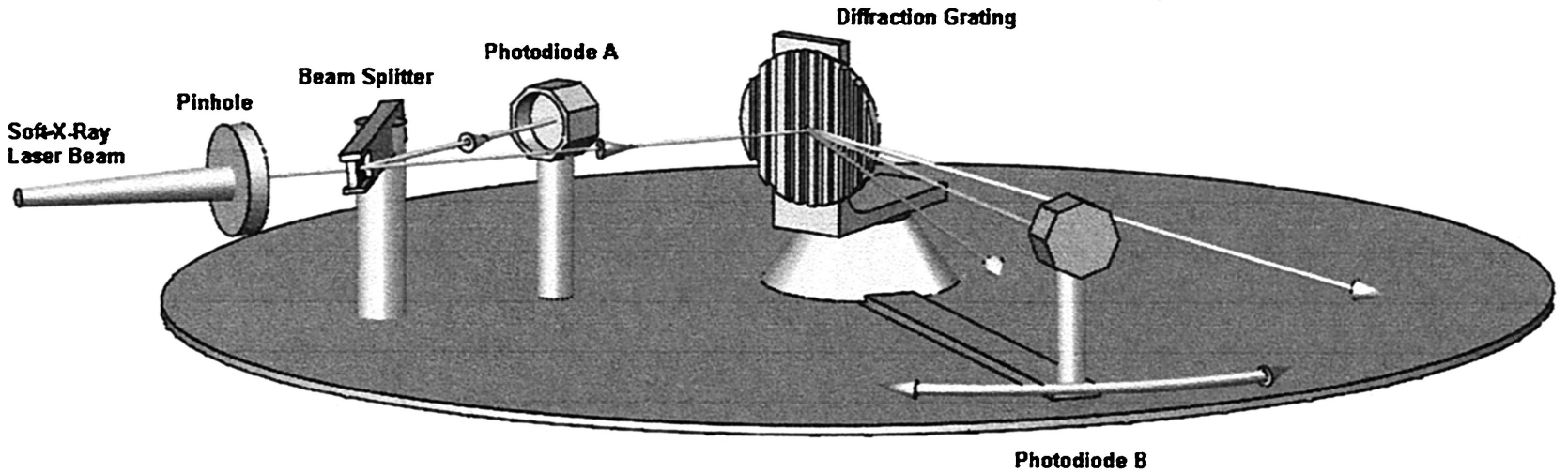

Fig. 2. Experimental setup used to characterize the efficiency of diffraction gratings at $46.9 \mathrm{~nm}$. Vacuum Photodiode B is rotated to measure the intensities of the various diffraction orders. Photodiode A is used to monitor the shot-to-shot variation of the intensity of the incident laser beam. 
trance of the chamber. With the objective of reducing the scattering of the data caused by shot-to-shot intensity variations of the laser, we normalized the intensity of the refracted beam to the intensity of the incident beam for each laser pulse. For this purpose we generated a reference signal by reflecting part of the incident laser beam with a 50\% transmissive gold-plated grid into a fixed reference vacuum photodiode (Photodiode A in Fig. 2). To obtain absolute values of the diffraction efficiency we calibrated the signal of the reference photodiode with respect to the intensity of the beam transmitted by the grid. We accomplished this calibration by removing Photodiode $\mathrm{B}$ from the lever arm and placing it in front of the grating. This calibration was repeated before and after each series of measurements. The photodiode signals were recorded and stored for every laser shot by a $500-\mathrm{MHz}$ analog bandwidth digital oscilloscope (Hewlett-Packard Model 54825A). The efficiency of each of the diffraction orders was measured by averaging of 30 laser shots.

The main contributor to the statistical error associated with the measurements is the electromagnetic noise produced by firing of the fast electrical discharge that generates the laser pulse, which affects the signal produced by the vacuum photodiodes. The standard deviation of several series of measurements (each consisting of 30 laser shots) of the zeroorder and first-order diffraction intensities taken for a fixed angular position of the grating was $\sim 10 \%$ of the mean value. All measurements fell within $\pm 13 \%$ of the mean. Possible sources of systematic error include saturation of the vacuum photodiode signal and failure of the active area of the detector to collect the entire laser beam when the detector is placed at a certain position. We checked the linearity of the detectors by measuring the response of the detectors as a function of applied voltage (increased voltage across the vacuum photodiodes decreased saturation by reducing the electron space charge). Based on these measurements, we placed thin-film aluminum filters at the inputs of the photodiodes and selected a sufficiently high-bias voltage to ensure their linearity to better than $5 \%$ over the entire range of intensities used in these measurements.

As was mentioned above, a pinhole was placed at the entrance of the measurement chamber to limit the beam diameter to $1 \mathrm{~mm}$. This ensured that we could detect the entire beam at all positions. Also, attention was paid to the digitization error introduced by the insufficiently fast sampling rate of the oscilloscope. To overcome this problem we slowed the photodiode signals, using a passive electronic circuit before digitization. The overall relative error of the measurements can be conservatively estimated to be less than $\pm 13 \%$.

\section{Simulation of the Grating Performance}

The classic formulation of the differential method used to simulate the grating performance ${ }^{9}$ is discussed below. To handle polarization effects, it is convenient to analyze two independent cases sepa- rately: that of an incident electric field parallel to the grooves ( $s, E_{\|}$, or TE polarization) and that of an incident magnetic field parallel to the grooves $\left(p, H_{\|}\right.$, or TM polarization). In the first case, the total (incident and diffracted) electric field is parallel to the grating grooves, whereas in the second case it is the total magnetic field that is parallel to the grating grooves. Taking into account that the differential method is well documented in the literature (see Ref. 9 and references therein) and that the main idea of its formulation is similar for both polarization cases, we give the relevant details for the $E_{\|}$case only. Recently, a new formulation of the differential method in TM polarization that removes numerical instabilities associated with deep gratings and highly reflecting materials was presented. ${ }^{11}$

We chose a rectangular coordinate system $(x, y, z)$ in such a way that the grating surface is described by the periodic function $y=g(x)=g(x+d)$, where $d$ is the period, with min $g(x)=0$ and $\max g(x)=h(h$ is the groove height). This surface separates two isotropic and homogeneous media, each one characterized by dielectric permittivity $\varepsilon_{j}(j=1,2)$. We assume that the magnetic permeability is everywhere equal to that of vacuum. The grooves of this grating lie along the $z$ axis, and the interface is illuminated from medium 1 by a monochromatic plane wave of frequency $\omega$. The plane of incidence (plane containing the wave vector of the incident wave and normal to the mean grating surface) coincides with the main section of the grating (plane perpendicular to the grating grooves). In this case, the wave vectors of the diffracted orders are all contained in the same plane. The components of $\mathbf{k}$ (the wave vector of the incident wave) can be written as $\mathbf{k}=\gamma_{0} \mathbf{x}+\beta_{0}$ $\mathbf{y}$, where $\gamma_{0}=k_{1} \sin \theta, \beta_{0}=k_{1} \cos \theta, \theta$ is the angle of incidence measured from the normal to the mean grating surface, $k_{1}=\varepsilon_{1} \omega / c$, and $c$ is the speed of light in vacuum.

The formulation of the method is based on the fact that, with the assumptions expressed above, the electromagnetic properties of the diffraction grating can be described by a coordinate-dependent dielectric permittivity $\varepsilon(x, y)$ with the following properties: (a) it is a real or a complex function; (b) it is independent of the $z$ coordinate; (c) it is constant and equal to $\varepsilon_{1}$ if $y>g(x)$; (d) it is constant and equal to $\varepsilon_{2}$ if $y<$ $g(x)$; and (e) for a fixed value of $y, 0<y<h$, function $\varepsilon(x, i)$ is periodic (period $d)$, piecewise, and continuous with respect to $x$.

Outside the modulated zone $0<y<h$, the dielectric permittivity is constant, and Maxwell's equations give the following analytical solutions for the total electric field $f(x, y)$ :

$$
\begin{array}{ll}
f(x, y)=f_{\text {inc }}(x, y)+\sum_{n} R_{n} \exp i\left(\gamma_{n} x+\beta_{n} y\right), & y>h, \\
f(x, y)=\sum_{n} T_{n} \exp i\left(\gamma_{n} x+\phi_{n} y\right), & y<0,
\end{array}
$$

where $f(x, y)$ represents the electric field in the $E_{\|}$ case or the magnetic field in the $H_{\|}$case, $f_{\text {inc }}$ corre- 
sponds to the incident field, $\gamma_{n}=\gamma_{o}+n 2 \pi / d, \beta_{n}=$ $\left(k_{1}{ }^{2}-\gamma_{n}{ }^{2}\right)^{1 / 2}, \phi_{n}=\left(k_{2}{ }^{2}-\gamma_{n}{ }^{2}\right)^{1 / 2}, k_{2}=\varepsilon_{2} \omega / c$, and $R_{n}$ and $T_{n}$ are the complex amplitudes of the reflected and transmitted diffracted orders, respectively.

In the modulated zone, Maxwell's equations give the following propagation equation for $f(x, y)$ :

$$
\nabla^{2} f(x, y)+\alpha(x, y) f(x, y)=0,
$$

where $\alpha(x, y)=(\omega / c)^{2} \varepsilon(x, y)$. The periodic nature of the grating allows us to expand the functions $\alpha(x, y)$ and $f(x, y)$ in the following manner:

$$
\begin{aligned}
& f(x, y)=\sum_{n} f_{n}(y) \exp i\left(\gamma_{n} x\right), \\
& \alpha(x, y)=\sum_{n} \alpha_{n}(y) \exp i(n 2 \pi x / d) .
\end{aligned}
$$

By introducing these expansions into propagation equation (1), we finally obtain a system of ordinary differential coupled equations for the unknown functions $f_{n}(y), n=-\infty \ldots \infty$ :

$$
f_{n}^{\prime \prime}=\alpha_{n} f_{n}-\sum_{n} \alpha_{n-m} f_{m} .
$$

Continuity of the electric field at $y=0$ and $y=h$ imposes four relations among $f_{n}^{\prime}(0), f_{n}(0), f_{n}^{\prime}(h)$, and $f_{n}(h)$ and the complex amplitudes $R_{n}$ and $T_{n}$. We can use two of these functions to integrate numerically the differential system in the interval $0<y<h$ :

$$
\begin{aligned}
f_{n}^{\prime}(0) & =-i \phi_{n} f_{n}(0), \\
f_{n}^{\prime}(h)-i \beta_{n} f_{n}(h) & =-2 i \beta_{0} \exp \left(-i \beta_{0} h\right) \delta_{0 n},
\end{aligned}
$$

and, after integration, the other two functions give the correct values of the complex amplitudes:

$$
\begin{aligned}
& R_{n}=f_{n}(h) \exp \left(-i \beta_{n} h\right)-\exp \left(-2 i \beta_{n} h\right) \delta_{0 n}, \\
& T_{n}=f_{n}(0)
\end{aligned}
$$

Finally, the efficiencies of the reflected and transmitted orders can be obtained as $r_{n}=\beta_{n}\left|R_{n}\right|^{2} / \beta_{0}$ and $t_{n}=$ $\phi_{n}\left|T_{n}\right|^{2} / \phi_{o}$, respectively.

To implement this differential formalism numerically we must assume that the field $f(x, y)$ is accurately represented by $N$ components of its series [from $n=-N_{-}$to $n=N_{+}$in Eq. (2), with $N=N_{-}+$ $\left.N_{+}+1\right]$, and truncate the infinite set of differential equations to order $N$. Standard algorithms, such as those of Runge-Kutta and Noumerov, can be used to integrate the system of Eq. (4). However, it should be noted that these algorithms start with known values of the function and its derivative at $y=0$ and proceed with the integration up to $y=h$ (or vice versa), but, in fact, these values are not known (we know only the incident field at $y=h$ ). To eliminate this difficulty we proceed in the following manner: First we find a basis for the space of solutions to system (4) together with condition (5) (the dimension of the space is $N$ ). Let us call the $n$th component of the $m$ th vector of this basis $f_{n}^{(m)}(y)\left(n, m=-N_{-}, \ldots\right.$, $\left.0, \ldots, N_{+}\right)$. To construct this basis, it is sufficient to integrate $N$ times system (4) together with condition (5), choosing $N$ linearly independent vectors $f^{(m)}(0)$, for example, $f_{n}^{(m)}(0)=\delta_{n m}$. Thus the desired solution to system (4) together with conditions (5) and (6) can be written in terms of this basis as

$$
f_{n}(y)=\sum_{n} S_{m} f_{n}^{(m)}(y)
$$

To find the constants $S_{m}$ we force this expression to satisfy relation (6), obtaining in this manner a linear system with $S_{m}$ as unknowns:

$$
\begin{aligned}
\sum_{n} S_{m}\left[f_{n}^{(m)^{\prime}}(h)-i \beta_{n} f_{n}^{(m)}(h)\right] & =-2 i \beta_{0} \\
& \times \exp \left(-i \beta_{0} h\right) \delta_{0 n} .
\end{aligned}
$$

Once this linear system has been inverted, we are ready to calculate the total electric field $f(x, y)$ everywhere. In particular, the complex amplitudes of the reflected orders $R_{n}$ can be calculated from Eq. (7).

The numerical code that we used to obtain the simulations presented in Section 4 was checked by use of several criteria such as power balance, reciprocity theorems, convergence and stability of the results with $N$, and length of the integration step. Furthermore, the results of the code for a large num-
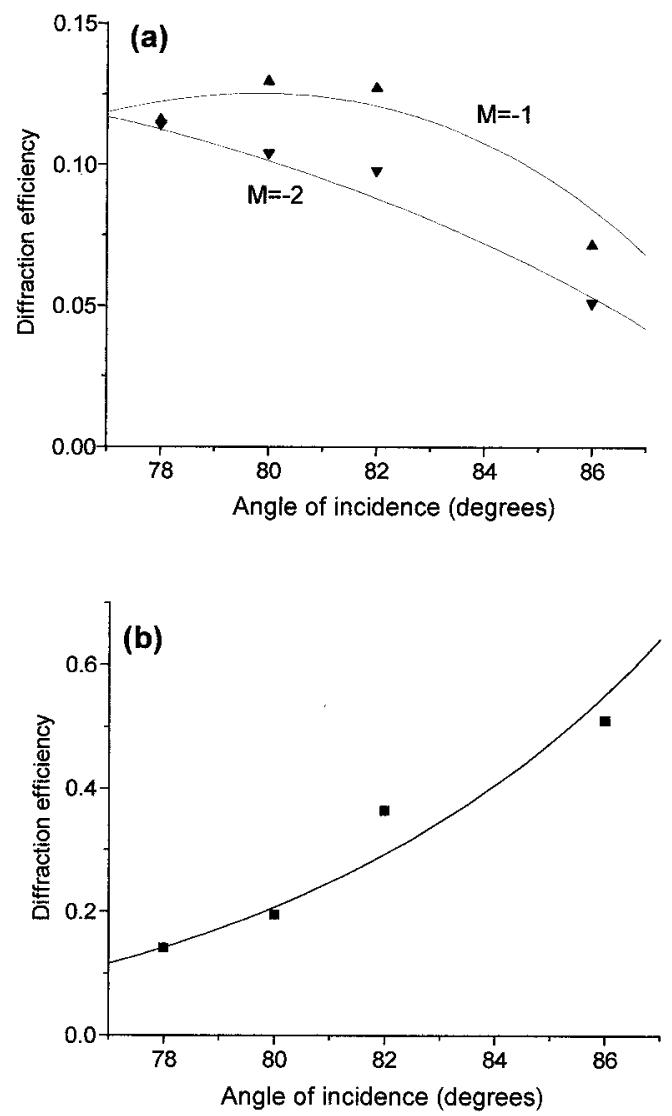

Fig. 3. Comparison of measured and computed absolute diffraction efficiencies of a 1800-line/mm gold-coated diffraction grating as a function of grazing-incidence angle. The angles between the facets and the mean surface are $A=10.028 \mathrm{deg}$ and $B=60 \mathrm{deg}$. (a) Data for first and second diffraction orders; (b) zero-order data. 


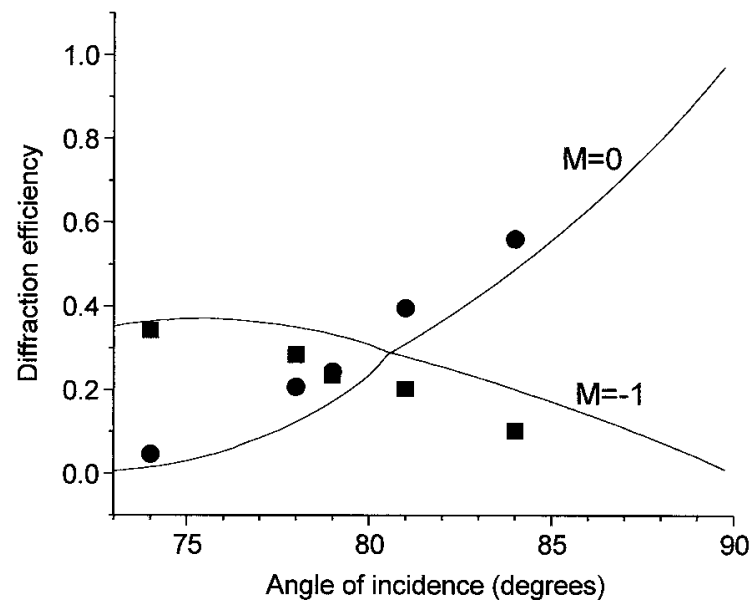

Fig. 4. Comparison of measured and computed diffraction efficiencies of the $M=0$ and $M=-1$ diffraction orders of a 300line/mm gold-coated diffraction grating as a function of incidence angle. The angles between the facets and the mean surface are $A$ $=1.33 \mathrm{deg}$ and $B=80 \mathrm{deg}$.

ber of grating configurations were compared with those given by other codes, based on widely different techniques, such as the Chandezon coordinate transformation method ${ }^{5}$ and the modal method developed by Li. ${ }^{6}$ Although we observed good agreement between these methods for gratings that support a number of diffracted orders (such as those used in the visible region of the electromagnetic spectrum) that is not too great, the differential method sketched above was the only one to produce stable results for gratings that support a large number of diffracted orders. Because of this particular capability, which has already been reported in the literature, ${ }^{9}$ we chose the differential method to simulate the performance of gratings designed for grazing-incidence operation in the soft-x-ray region. The wavelength-to-period ratios for the gratings considered here are $\lambda / d=$ 0.01407 (300 lines $/ \mathrm{mm})$ and $\lambda / d=0.08442(1800$ lines $/ \mathrm{mm}$ ), which give a total of 143 and 24 propagating orders, respectively. Although propagating and nonpropagating orders are both, in principle, fundamental to representing the correct field, the number of propagating orders can be used as a first estimation for the numerical parameters $N, N_{-}$, and $N_{+}$. We chose the final values for these parameters by considering convergence and stability criteria. Note that gratings with large numbers of propagating orders can usually be excessively demanding for any theoretical method. This is so because the diminishing exponential terms associated with evanescent orders can lead to bad conditioning of the $N \times N$ matrix to be inverted [Eq. (10)]. Considering that our code is almost at the limit of its possibilities for values of $N$ beyond $\sim 170$, for the 300 -line/mm grating we were not able to include many nonpropagating orders in the truncated series. However, we have observed that better-conditioned matrices with convergence of the results up to $10^{-2}$ are obtained when not all the propagating orders are included. This
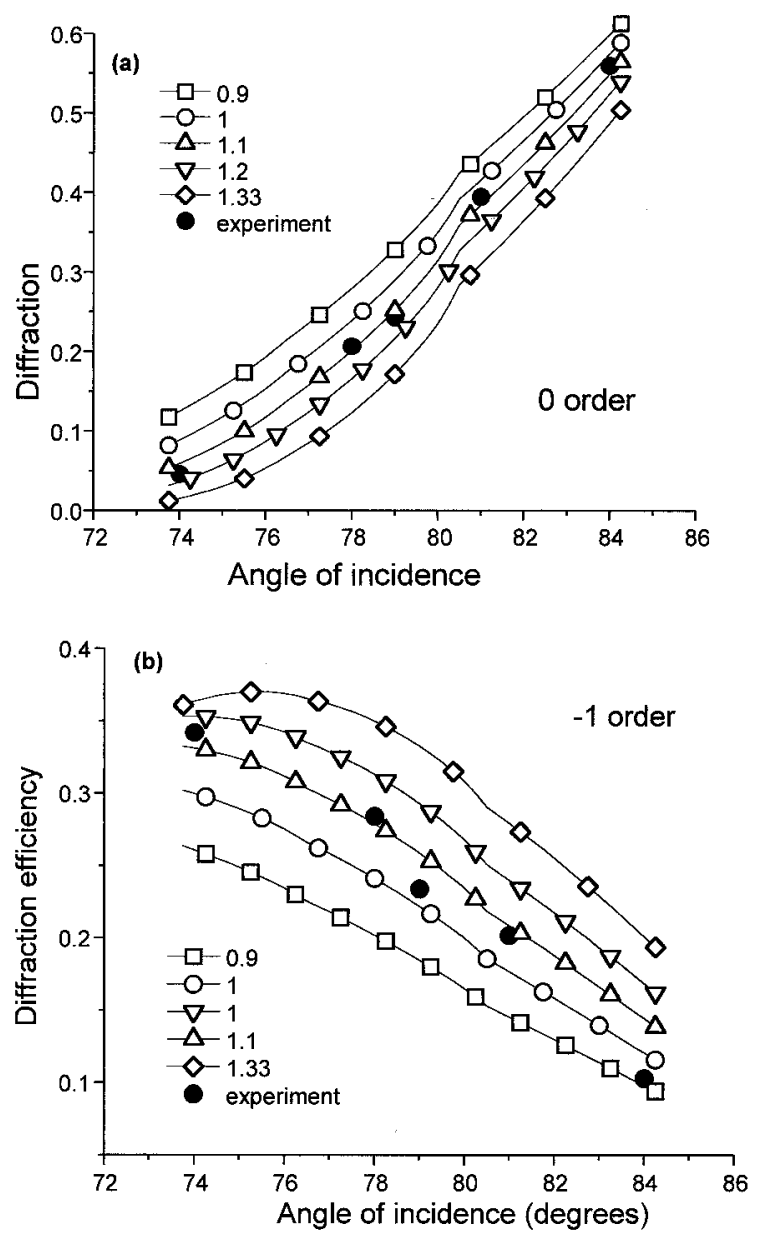

Fig. 5. Comparison of measured and computed diffraction efficiencies of the $300-$ line $/ \mathrm{mm}$ gold-coated diffraction grating as a function of incidence angle and for several values of angle $B$. The blaze angle is $1.33 \mathrm{deg}$.

behavior of the differential method, which is not generally valid and could seem counterintuitive, could be a consequence of the fact that for the $300-$ line $/ \mathrm{mm}$ grating most of the diffracted power is concentrated about the zero order.

\section{Comparison of Measurements and Simulations}

In our calculations we have assumed ideal triangular profiles. For the angles $A$ and $B$ between the groove facets and the mean grating surface we used the values $A=10.028 \mathrm{deg}$ and $B=60 \mathrm{deg}$ for the 1800 line $/ \mathrm{mm}$ grating and $A=1.33 \mathrm{deg}$ and $B=80 \mathrm{deg}$ for the 300 -line $/ \mathrm{mm}$ grating.

Figure 3 compares the measured diffraction efficiency of the 1800 -line/mm grating with the model simulation results for incidence angles of 78-86 deg. The refractive index of gold was chosen as $v=0.89+$ $0.07 i$. The measured efficiency of the first and second diffraction orders $(M=-1, M=-2)$ is marked by triangles in Fig. 3(a). The curves represent the results of the simulations. Figure 3(b) shows the results for order $0(M=0)$. Excellent agreement is observed between measurements and theory for all 
the diffraction orders over the entire range of incidence angles. The measured absolute diffraction efficiency values for the first and second diffraction orders are within $1.5 \%$ of the computed values.

The results for the 300 -line/mm grating are shown in Fig. 4. The measurements cover angles of incidence of 74-84 deg. The agreement between the measured and computed values is not so good as for the $1800-$ line $/ \mathrm{mm}$ grating. The simulation overestimates the absolute diffraction efficiency of the first order by as much as $8.5 \%$. To illustrate the effect of possible departures from the ideal profile, we repeated the calculations for the $300-$ line $/ \mathrm{mm}$ triangular grating but used different values for angles $A$ and $B$ in the vicinity of the nominal values given above. We observed that, for the angles of incidence considered, the efficiencies remain almost unchanged when angle $B$ is varied from 71 to $89 \mathrm{deg}$ and angle $A$ remains fixed at the nominal value 1.33. The calculations, however, depend much more on angle $A$ (blaze angle), which in the manufacturing process has been controlled within a $10 \%$ error. The influence of this shape variation is illustrated in Fig. 5, where we compare the experimental results with those obtained for various values of angle $B(0.9,1.0$, 1.1 , and $1.2 \mathrm{deg}$ ).

As was discussed in Section 3, the poorer agreement in this case could be due to the large number of modes supported by the 300 -line/mm grating, which approaches the limit of the capabilities of the present code, or to an overestimation of angle $B$.

\section{Conclusions}

In summary, we have used a tabletop laser to measure the efficiency with which gratings diffract 46.9 $\mathrm{nm}$ radiation. The measurements were compared with the results of an electromagnetic code based on the classic formulation of the differential method. The measurements indicate that the code accurately predicts grating performance when the number of propagating modes is relatively small (e.g., 24 propagating modes, 1800 -line/mm grating). When the number of propagating modes is large (e.g., 143 propagating modes, $300-$ line $/ \mathrm{mm}$ grating) the code is less accurate. The results illustrate the significant potential of tabletop soft-x-ray lasers as a new powerful characterization tool for short-wavelength optics.
We gratefully acknowledge the support of the Colorado Photonics and Optoelectronics Program and of the National Science Foundation and a grant from the W. M. Keck Foundation. R. A. Depine acknowledges the support of the Universidad de Buenos Aires and of the Agencia Nacional de Promoción Científica y Tecnológica (BID 802/OC-AR03-04457). We thank B. R. Benware and T. Lescher for their contributions to the measurements and A. Vinogradov and I. Artiukov for useful discussions.

\section{References}

1. D. T. Attwood, P. Naulleau, K. A. Goldberg, E. Tejnil, C. Chang, R. Beguiristain, P. Batson, J. Bokor, E. Gullokson, M. Koike, H. Medecki, and J. Underwood. "Tunable coherent radiation in the soft x-ray and extreme ultraviolet spectral regions," IEEE J. Quantum Electron. 35, 709-720 (1999).

2. A. Rudquist, C. G. Durfee III, Z. Chang, C. Herne, S. Backus, M. Murnane, and H. C. Kapteyn. "Phase-matched generation of coherent soft x-rays," Science 280, 1412-1415 (1998).

3. J. Filevich, K. Kanizay, M. C. Marconi, J. L. A. Chilla, and J. J. Rocca, "Dense plasma diagnostics with an amplitude-division soft-x-ray laser interferometer based on diffraction gratings," Opt. Lett. 25, 356-358 (2000)

4. H. Medecki, E. Tejnil, K. A. Goldberg, and J. Bokor, "Phaseshifting point diffraction interferometer," Opt. Lett. 21, 15261528 (1996)

5. J. Chandezon, M. T. Dupuis, G. Cornet, and D. Maystre, "Multicoated gratings: a differential formalism applicable in the entire optical region," J. Opt. Soc. Am. A 72, 839-846 (1982).

6. L. Li, "Multilayer modal method for diffraction gratings of arbitrary profile, depth, and permittivity,” J. Opt. Soc. Am. A 10, 2581-2591 (1993).

7. B. R. Benware, C. D. Macchietto, C. H. Moreno, and J. J. Rocca, "Demonstration of a high average power tabletop soft x-ray laser," Phys. Rev. Lett. 81, 5804-5807 (1998).

8. C. D. Macchietto, B. R. Benware, and J. J. Rocca. "Generation of millijoule-level soft-x-ray laser pulses at $4-\mathrm{Hz}$ repetition rate in a highly saturated tabletop capillary discharge amplifier," Opt. Lett. 24, 1115-1117 (1999).

9. P. Vincent, "Differential methods," in Electromagnetic Theory of Gratings, R. Petit, ed. (Springer-Verlag, Berlin, 1980), Chap. 4.

10. J. J. Rocca, D. P. Clark, J. L. A. Chilla, and V. N. Shlyaptsev, "Energy extraction and achievement of the saturation limit in a discharge pumped table-top soft x-ray amplifier," Phys. Rev. Lett. 77, 1476-1479 (1996).

11. E. Popov and M. Nevière, "Differential theory for diffraction gratings: a new formulation for TM polarization with rapid convergence," Opt. Lett. 25, 598-600 (2000). 